\title{
Introduction to the 35th International Conference on Logic Programming Special Issue
}

\author{
ESRA ERDEM \\ Sabanci University, Turkey \\ (e-mail: esraerdem@sabanciuniv.edu) \\ ANDREA FORMISANO \\ Università di Perugia, Italy \\ (e-mail: andrea.formisano@unipg.it) \\ GERMÁN VIDAL \\ MiST, VRAIN, Universitat Politècnica de València, Spain \\ (e-mail: gvidal@dsic.upv.es) \\ FANGKAI YANG \\ NVIDIA Corporation, USA \\ (e-mail: fangkaiy@nvidia.com)
}

submitted 09 August 2019; accepted 14 August 2019

This volume contains the regular papers of the 35th International Conference on Logic Programming (ICLP 2019), held in Las Cruces, New Mexico, USA, from September 20 to September 25, 2019.

Since the first conference held in Marseille in 1982, ICLP has been the premier international event for presenting research in logic programming. The scope of the conference covers all areas of logic programming including:

Foundations: Semantics, Formalisms, Nonmonotonic reasoning, Knowledge representation.

Languages: Concurrency, Objects, Coordination, Mobility, Higher Order, Types, Modes, Assertions, Modules, Meta-programming, Logic-based domain-specific languages, Programming Techniques.

Declarative programming: Declarative program development, Analysis, Type and mode inference, Partial evaluation, Abstract interpretation, Transformation, Validation, Verification, Debugging, Profiling, Testing, Execution visualization

Implementation: Virtual machines, Compilation, Memory management, Parallel or distributed execution, Constraint handling rules, Tabling, Foreign interfaces, User interfaces.

Related Paradigms and Synergies: Inductive and Co-inductive Logic Programming, Constraint Logic Programming, Answer Set Programming, Interaction with SAT, SMT and CSP solvers, Logic programming techniques for type inference and theorem proving, Argumentation, Probabilistic Logic Programming, Relations to object-oriented and Functional programming.

Applications: Databases, Big Data, Data integration and federation, Software engineering, Natural language processing, Web and Semantic Web, Agents, Artificial intelligence, Computational life sciences, Education, Cybersecurity, and Robotics. 
Besides the main track, ICLP 2019 included the following additional tracks and special sessions:

- Applications Track: This track invited submissions of papers on emerging and deployed applications of logic programming, describing all aspects of the development, deployment, and evaluation of logic programming systems to solve real-world problems, including interesting case studies and benchmarks, and discussing lessons learned.

- Sister Conferences and Journal Presentation Track: This track provided a forum to discuss important results related to logic programming that appeared recently (from January 2017 onwards) in selective journals and conferences, but have not been previously presented at ICLP.

- Research Challenges in Logic Programming Track: This track invited submissions of papers describing research challenges that an individual researcher or a research group is currently attacking. The goal of the track is to promote discussions, exchange of ideas, and possibly stimulate new collaborations.

- Special Session. Women in Logic Programming: This special session included an invited talk and presentations describing research by women in logic programming.

The organizers of ICLP 2019 were:

\section{General Chairs}

Enrico Pontelli, New Mexico State University

Tran Cao Son, New Mexico State University

\section{Program Chairs}

Esra Erdem, Sabanci University

Germán Vidal, Universitat Politècnica de València

\section{Publicity Chair}

Ferdinando Fioretto, Georgia Institute of Technology

\section{Workshops Chair}

Martin Gebser, University of Klagenfurt and Graz University of Technology

\section{Tutorials Chair}

Pedro Cabalar, University of Corunna

\section{Chairs}

Paul Fodor, Stony Brook New York

Daniela Inclezan, Miami University

\section{Programming Competition Chairs}

José Morales, IMDEA Software Institute

Orkunt Sabuncu, TED University

\section{Applications Track Chairs}

Andrea Formisano, Università di Perugia

Fangkai Yang, NVIDIA Corporation 


\section{Sister Conferences and Journal Presentation Track Chairs}

Bart Bogaerts, Vrije Universiteit Brussel

Giovambattista Ianni, Università della Calabria

\section{Research Challenges in Logic Programming Track Chairs}

Alessandro dal Palù, Università di Parma

Amelia Harrison, University of Texas at Austin and Google Inc.

Joohyung Lee, Arizona State University

\section{Women in Logic Programming Special Session Chairs}

Alicia Villanueva, Universitat Politècnica de València

Marina De Vos, University of Bath

Three kinds of submissions were accepted:

- Technical papers for technically sound, innovative ideas that can advance the state of logic programming.

- Application papers that impact interesting application domains.

- System and tool papers which emphasize novelty, practicality, usability, and availability of the systems and tools described.

ICLP implemented the hybrid publication model used in all recent editions of the conference, with journal papers and Technical Communications (TCs), following a decision made in 2010 by the Association for Logic Programming. Papers of the highest quality were selected to be published as rapid publications in this special issue of TPLP. The TCs comprise papers which the Program Committee (PC) judged of good quality but not yet of the standard required to be accepted and published in TPLP as well as extended abstracts from the different tracks and dissertation project descriptions stemming from the Doctoral Program (DP) held with ICLP.

We have received 122 submissions of abstracts, of which 92 resulted in full submissions, distributed as follows: ICLP main track (59), Applications track (9 full papers and 9 short papers), Sister Conferences and Journal Presentation track (11), and Women in Logic Programming session (4). The Program Chairs organized the refereeing process, which was undertaken by the PC with the support of external reviewers. Each technical paper was reviewed by at least three referees who provided detailed written evaluations. This yielded submissions short-listed as candidates for rapid communication. The authors of these papers revised their submissions in light of the reviewers suggestions, and all these papers were subject to a second round of reviewing. Of these candidates papers, 30 were accepted as rapid communications, to appear in the special issue. In addition, the PC recommended 45 papers to be accepted as technical communications, to appear at Electronic Proceedings in Theoretical Computer Science (EPTCS) either as full papers or extended abstracts, of which 44 were also presented at the conference ( 1 was withdrawn).

The 30 rapid communications that appear in this special issue are listed below:

- Tiantian Gao, Paul Fodor and Michael Kifer. Querying Knowledge via Multi-Hop English Questions.

- Mario Alviano, Nicola Leone, Pierfrancesco Veltri and Jessica Zangari. Enhancing magic sets with an application to ontological reasoning. 
- Jorge Fandinno. Founded (Auto)Epistemic Equilibrium Logic Satisfies Epistemic Splitting.

- Giovanni Amendola and Francesco Ricca. Paracoherent Answer Set Semantics meets Argumentation Frameworks.

- Giovanni Amendola, Francesco Ricca and Mirek Truszczynski. Beyond NP: Quantifying over Answer Sets.

- Elvira Albert, Miquel Bofill, Cristina Borralleras, Enrique Martin-Martin and Albert Rubio. Resource Analysis driven by (Conditional) Termination Proofs.

- Giovanni Amendola, Carmine Dodaro and Marco Maratea. Abstract Solvers for Computing Cautious Consequences of ASP programs.

- Giovanni Amendola, Carmine Dodaro and Francesco Ricca. Better Paracoherent Answer Sets with Less Resources.

- Gonzague Yernaux and Wim Vanhoof. Anti-unification in Constraint Logic Programming.

- Stefania Costantini. About epistemic negation and world views in Epistemic Logic Programs.

- Fernando Sáenz-Pérez. Applying Constraint Logic Programming to SQL Semantic Analysis.

- Wolfgang Faber, Michael Morak and Stefan Woltran. On Uniform Equivalence of Epistemic Logic Programs.

- Efthimis Tsilionis, Nikolaos Koutroumanis, Panagiotis Nikitopoulos, Christos Doulkeridis and Alexander Artikis. Online Event Recognition from Moving Vehicles: Application Paper.

- Bernardo Cuteri, Carmine Dodaro, Francesco Ricca and Peter Schüller. Partial Compilation of ASP Programs.

- María Alpuente, Demis Ballis, Santiago Escobar and Julia Sapiña. Symbolic Analysis of Maude Theories with Narval.

- Mario Alviano, Carmine Dodaro, Johannes K. Fichte, Markus Hecher, Tobias Philipp and Jakob Rath. Inconsistency Proofs for ASP: The ASP-DRUPE Format.

- Felicidad Aguado, Pedro Cabalar, Jorge Fandinno, David Pearce, Gilberto Perez and Concepcion Vidal. Revisiting Explicit Negation in Answer Set Programming.

- Angelos Charalambidis, Christos Nomikos and Panos Rondogiannis. The Expressive Power of Higher-Order Datalog.

- Joao Alcantara, Samy Sá and Juan Carlos Acosta-Guadarrama. On the Equivalence Between Abstract Dialectical Frameworks and Logic Programs.

- Francesco Calimeri, Giovambattista Ianni, Francesco Pacenza, Simona Perri and Jessica Zangari. Incremental Answer Set Programming with Overgrounding.

- Thomas Eiter, Paul Ogris and Konstantin Schekotihin. A Distributed Approach to LARS Stream Reasoning (System paper).

- Jesús J. Domnech, John Gallagher and Samir Genaim. Control-Flow Refinement by Partial Evaluation, and its Application to Termination and Cost Analysis.

- Amelia Harrison and Vladimir Lifschitz. Relating Two Dialects of Answer Set Programming.

- Arpit Sharma. Using Answer Set Programming for Commonsense Reasoning in the Winograd Schema Challenge.

- Matti Berthold, Ricardo Gonçalves, Matthias Knorr and Joao Leite. A Syntactic Operator for Forgetting that Satisfies Strong Persistence. 
- Ariyam Das and Carlo Zaniolo. A Case for Stale Synchronous Distributed Model for Declarative Recursive Computation.

- Alessio Fiorentino, Nicola Leone, Marco Manna, Simona Perri and Jessica Zangari. Precomputing Datalog evaluation plans in large-scale scenarios.

- Yi Wang, Shiqi Zhang and Joohyung Lee. Bridging Commonsense Reasoning and Probabilistic Planning via a Probabilistic Action Language.

- Joaquin Arias and Manuel Carro. Evaluation of the Implementation of an Abstract Interpretation Algorithm using Tabled CLP.

- David Spies, Jia-Huai You and Ryan Hayward. Domain-Independent Cost-Optimal Planning in ASP.

In addition to the presentations of accepted papers, the technical program of ICLP 2019 included four invited talks:

- Adnan Darwiche. What Logic Can Do for AI Today.

- Nicola Leone. ASP Applications for AI and Industry.

- Sheila McIlraith. Reward Machines: Structuring reward function specifications and reducing sample complexity in reinforcement learning.

- Yuliya Lierler. System PROJECTOR: An Automatic Program Rewriting Tool for Non-Ground Answer Set Programs.

and three invited tutorials:

- Chitta Baral. Knowledge Representation and Reasoning issues in Natural Language Question Answering.

- Serdar Kadioglu. Constraint Programming for Resource Management.

- Guy Van den Broeck. Tractable Probabilistic Circuits

Furthermore, after a thorough examination of citation indices (e.g., Web of Science, Google Scholar), two test-of-time awards were identified:

- The John Alan Robinson 20 year test of time award: Frédéric Benhamou, Frédéric Goualard, Laurent Granvilliers, Jean-Francois Puget. Revising Hull and Box Consistency. ICLP 1999: 230-244

- The Alain Colmerauer 10 year test of time award: Martin Gebser, Max Ostrowski, Torsten Schaub. Constraint Answer Set Solving. ICLP 2009: 235-249

We are deeply indebted to the Program Committee members and external reviewers, as the conference would not have been possible without their dedicated, enthusiastic and outstanding work. The Program Committee members of ICLP 2019 were: 


Hassan Ait-Kaci
Rachel Ben-Eliyahu-Zohary
Pedro Cabalar
Marina De Vos
Wolfgang Faber
John Gallagher
Michael Hanus
Giovambattista Ianni
Tomi Janhunen
Vladimir Lifschitz
Nicola Leone
Jose F. Morales
Magdalena Ortiz
Simona Perri
Alessandra Russo
Torsten Schaub
Francesca Toni
Alicia Villanueva
Stefan Woltran
Jia-Huai You

Mario Alviano
Bart Bogaerts
Michael Codish
Agostino Dovier
Fabio Fioravanti
Martin Gebser
Amelia Harrison
Daniela Inclezan
Angelika Kimmig
Evelina Lamma
Yanhong Annie Liu
Emilia Oikarinen
Mauricio Osorio
Enrico Pontelli
Orkunt Sabuncu
Guillermo R. Simari
Paolo Torroni
Kewen Wang
Fangkai Yang
Zhizheng Zhang

Roman Bartak

Gerhard Brewka

Stefania Costantini

Thomas Eiter

Andrea Formisano

Michael Gelfond

Manuel Hermenegildo

Katsumi Inoue

Ekaterina Komendantskaya

Joohyung Lee

Fred Mesnard

Carlos Olarte

Barry O'Sullivan

Ricardo Rocha

Chiaki Sakama

Theresa Swift

Tran Cao Son

Jan Wielemaker

Roland Yap

The Program Committee members of the Applications Track were:

$\begin{array}{llll}\text { Chitta Baral } & \text { Alex Brik } & \text { Francesco Calimeri } & \text { Xiaoping Chen } \\ \text { Federico Chesani } & \text { Martín Diéguez } & \text { Gerhard Friedrich } & \text { Gopal Gupta } \\ \text { Jianmin Ji } & \text { Gabriele Kern-Isberner } & \text { Zeynep Kiziltan } & \text { Viviana Mascardi } \\ \text { Yunsong Meng } & \text { Francesco Ricca } & \text { Mohan Sridharan } & \text { David Warren } \\ \text { Shiqi Zhang } & \text { Neng-Fa Zhou } & & \end{array}$

The Program Committee members of the Special Session: Women in Logic Programming were:
Elvira Albert
Stefania Costantini
Ines Dutra
Daniela Inclezan
Ekaterina Komendantskaya
Simona Perri
Francesca Toni

The external reviewers were:

$\begin{array}{ll}\text { Van Nguyen } & \text { Bernardo Cuteri } \\ \text { Anna Schuhmann } & \text { Alberto Policriti } \\ \text { Vítor Santos Costa } & \text { Arash Karimi } \\ \text { Michael Frank } & \text { Roland Kaminski } \\ \text { Jose Luis Carballido } & \text { Christopher Kane } \\ \text { Isabel Garcia-Contreras } & \text { José Abel Castellanos Joo } \\ \text { Vitaly Lagoon } & \text { Jannik Dreier } \\ \text { Marco Gavanelli } & \text { Emanuel Sallinger } \\ \text { Wanwan Ren } & \text { Kinjal Basu } \\ \text { Marco Alberti } & \text { Gianluca Amato } \\ \text { Joaquin Arias } & \text { Miguel Areias } \\ \text { Farhad Shakerin } & \text { Nada Sharaf } \\ \text { Yuanlin Zhang } & \text { Yi Tong } \\ \text { Saksham Chand } & \text { Yan Zhang }\end{array}$
Dennis Dams
Jessica Zangari
Joxan Jaffar
Javier Romero
Emanuele De Angelis
Wolfgang Dvorak
Philipp Wanko
Weronika T. Adrian
Patrick Kahl
Juan Carlos Nieves
Konstantin Schekotihin
Christoph Redl
K. Tuncay Tekle
Jessica Zangari

We would also like to express our gratitude to the full ICLP 2019 organization committee. Our gratitude must be extended to Torsten Schaub, who is serving in the role of President of the Association of Logic Programming (ALP), to all the members of the ALP Executive Committee and to Mirek Truszczynski, Editor-in-Chief of TPLP. Also, to the staff at Cambridge University Press for their assistance. Finally, we wish to thank each author of every submitted paper, since their efforts keep the conference alive and the participants to ICLP for bringing and sharing their ideas and latest developments. 